\title{
EDITORIAL
}

\section{Predicting preterm birth: where do the major challenges lie?}

\section{Journal of Perinatology (2012) 32, 481-482; doi:10.1038/jp.2012.54}

The cost of prematurity, accounted for in both human currency and financial dollars, has fueled decades of research committed to developing models with the ability to predict preterm birth (PTB). The goal of these models is either to improve outcomes for preterm infants through optimizing care or to reduce the PTB rate itself. This field of research has generated contributions to our understanding of the mechanisms responsible for preterm labor and even a few clinical achievements: progesterone to prevent recurrent PTB, antenatal corticosteroids (ACS) to improve a host of neonatal outcomes and cerclage in patients with multiple prior losses consistent with cervical insufficiency. ${ }^{1-3}$ However, an overview of the literature demonstrates far more failures than successes.

In the United States, PTB remains above 12\% despite a dizzying array of tools for predicting PTB, including risk assessment, cervical length ultrasound, uterine contraction monitoring, fetal fibronectin, salivary estriol, and endless permutations of biomarkers X, Y and Z. ${ }^{4}$ None of these 'prediction' algorithms has sufficient accuracy to be clinically useful. In the current edition of the Journal of Perinatology, Ruiz et al. ${ }^{5}$ describe associations between cytokines and cytokine receptors in maternal plasma at 22 to 24 weeks gestation and subsequent PTB. Their finding that increased expression of interleukin-1 receptor antagonist (IL-1ra) in maternal plasma is associated with PTB is an important contribution to our understanding of how maternal cytokine cascade is activated prior to PTB, particularly with early PTB prior to 32 weeks gestation. However, the authors' published odds ratios for cytokine markers are between 2.0 and 2.5, which are similar to previous biomarker publications, and are not likely to be clinically useful as highlighted in a recent review by Menon et al. ${ }^{6}$ in Reproductive Sciences.

As a perinatal research community we have been reduced to declaring victory by adopting algorithms that save money by avoiding interventions without improving any health outcomes. So where do the major challenges lie? If significant reductions in the PTB rate appear to be unlikely in the short term, then is the development of an accurate prediction model a more achievable goal? Further, how would such a model improve perinatal outcomes? To address the possible benefits of a PTB prediction model, we analyzed our performance at Oregon Health \& Science University. At our center, among a cohort of patients with singleton pregnancies presenting with preterm labor, only $24 \%$ received ACS in an optimal treatment window of $2-14$ days before delivery. ${ }^{7}$
A more strict definition of optimal ACS timing (2-7 days) reduced this percentage to $19 \%$. Overall, less than $50 \%$ of patients who delivered $<34$ weeks gestation received ACS in the optimal treatment window and more than one-third of patients receiving ACS delivered after 37 weeks gestation. We should be able to do better. Even a 'poor' model with a predictive accuracy of 50\% would double the percentage of fetuses exposed to ACS within optimal timing and this would lead to significant improvements in neonatal outcomes without affecting the PTB rate at all.

Hence, with the focus not on reduction in PTB, but on developing an improved prediction model, we identify three focus areas for prematurity research and discuss each briefly: (1) differentiating early from late PTB, (2) etiology-specific approaches to prediction and (3) time-from-delivery analysis.

(1) Differentiating early from late PTB: Prediction of PTB should be dichotomized into the prediction of two separate events - early PTB before 34 weeks gestation and late PTB (34-37 weeks gestation). Early PTB represents $3.6 \%$ of US births, but accounts for the majority of neonatal mortality. ${ }^{8}$ Despite overwhelming data that at least $80 \%$ of early PTB is caused by infection of the choriodecidua and amniotic cavity, ${ }^{9,10}$ the genetic contributions and protein pathways resulting in early PTB have not been well characterized. On the contrary, PTB $>34$ weeks gestation is rarely associated with infection or inflammation, and the distribution of late PTB and post-term pregnancy are tails of a normal distribution, suggesting that these two entities are extremes of a normal physiological process. These two entities should be thought of as different pathological conditions; one prediction model will not work for both.

(2) Etiology-specific approaches to prediction: Within this framework, the work published by Ruiz et al. may represent a significant step in the right direction. A model to predict infectionmediated PTB will be useful in predicting early PTB and, in the absence of a clear understanding of other causes of early PTB, is the most viable approach for research. A follow-up study examining how IL-1ra, IL-10 and other cytokines (TNF $\alpha$, MMP-9 and IL-6) can be utilized to predict infection-mediated PTB may have immediate clinical benefits. Although treatment of infectionmediated PTB may be an achievable long-term goal, ACS administration has already been shown to reduce mortality, neurologic handicap and chronic lung disease by 50 to $70 \%$ in premature infants born before 34 weeks gestation, ${ }^{2,11}$ even in the setting of intra-amniotic infection and inflammation. ${ }^{12}$ As our 
understanding of how other pathological pathways may result in PTB increases, a long-term goal of research should be to develop a multi-analyte tool not only to predict PTB but also to identify the specific etiology involved, which may lead to more effective efforts to reduce the overall PTB rate.

(3) Time-from-delivery analysis: As a final comment, we point out that, historically, scientists have understood that gene expression varies across different gestational age windows, and that predictive tests function optimally by restricting prediction to a narrow gestational age window. Successful examples of this approach include the maternal marker test (commonly called the sequential screen or quadruple screen), which can predict Down syndrome and Edwards syndrome in pregnancy by analysis of maternal blood. The quadruple screen, for instance, works well within a narrow gestational age window of 16-20 weeks, and normal protein levels vary by each week of gestation. Diagnosis of ectopic pregnancy, gestational trophoblastic cancers and even a simple urine pregnancy test are other successful examples of this fundamental doctrine of medical prediction during pregnancy. This same methodology has been applied to predicting PTB, but with extremely poor results. The review by Menon ${ }^{6}$ cited previously concludes that none of the published biomarkers to date have the predictive accuracy necessary to be recommended for clinical practice.

Prediction of PTB differs from detection of Down syndrome or ectopic pregnancies. These conditions are uniformly present during the entire length of gestation. Simply put, embryos don't develop from a normal karyotype to Down syndrome and pregnancies don't convert from intra-uterine implantation to ectopic implantation. On the contrary, we hypothesize that early PTB is caused by an infection that occurs after normal pregnancy implantation and is highly variable in timing. It is likely that prediction of early PTB by a standard approach (comparison of protein levels at distinct gestational ages) is not achievable. Not all patients who deliver at 30 weeks gestation will have early infection at 20,24 or 28 weeks, so protein analysis at those gestational ages will always suffer from that limitation. An alternate approach would be to analyze biological specimens classified in relation to time from actual delivery. For example, if a patient delivers at 29 0/7 weeks gestation, a sample taken at 28 0/7 weeks gestation could be classified as ' $t-7$ ' and grouped with other ' $t-7$ ' samples for composite analysis. A comparative analysis of samples taken between ' $\mathrm{t}-2$ ' and ' $\mathrm{t}-\mathrm{7}$ ' may display quantitative protein differences that are not evident among samples taken between 20-22 weeks gestation to predict a birth that occurs either 40 or 100 days later. This type of analysis could be performed using bio-libraries already in existence.

Although accurate prediction of PTB has remained elusive for decades, we feel that the following three areas of focus are useful: (1) differentiating early from late PTB, (2) etiology-specific approaches to prediction and (3) appropriate analysis timing of samples. Hopefully, with these and other insights into PTB, the current decade will be the one in which accurate prediction of PTB becomes clinically available.

\section{Conflict of interest}

The authors declare no conflict of interest.

L Pereira and AB Caughey
Department of Obstetrics and Gynecology; Oregon Health \&
Science University, Portland, OR, USA
E-mail: pereiral@obsu.edu or caughey@obsu.edu

\section{References}

1 Meis PJ, Klebanoff M, Thom E, Dombrowski MP, Sibai B, Maowad AL et al. Prevention of recurrent preterm delivery by 17 alpha-hydroxyprogesterone caproate. $N$ Engl J Med 2003; 349(13): 1299.

2 Crowley P. Prophylactic corticosteroids for preterm birth. Cochrane Pregnancy and Childbirth Group. Cochrane Database of Syst Rev 2006; 3: 00075320-10000000000280 .

3 Berghella V, Rafael TJ, Szychowski JM, Rust OA, Owen J. Cerclage for short cervix on ultrasonography in women with singleton gestations and previous preterm birth: a meta-analysis. Obstet Gynecol 2011; 117(3): 663-671.

4 Moawad AH, Goldenberg RL, Mercer B, Meis PJ, Iams JD, Das A et al. The Preterm Prediction Study: the value of serum alkaline phosphatase, alpha-fetoprotein, plasma corticotropin releasing hormone, and other serum markers for the prediction of spontaneous preterm birth. Am J Obstet Gynecol 2002; 186(5): 990-996.

5 Ruiz RJ, Jallo N, Murphey C, Marti CN, Godbold E, Pickler E. Second trimester maternal plasma levels of Cytokines IL-1Ra, IL-6, and IL-10 and preterm birth. J Perinatol 2012.

6 Menon R, Torloni MR, Voltolini C, Torricelli M, Merialdi M, Betran AP et al. Biomarkers of spontaneous preterm birth: an overview of the literature in the last four decades. Reprod Sci 2011; 18(11): 1046-1070.

7 Tronnes A, Kinhnarath A, Caughey AB, Snyder J, Pereira L. Preterm birth and antenatal corticosteroid administration: how good are we at achieving optimal timing? Reprod Sci Suppl 2012; 19(3): 231A.

8 Martin JA. Centers for Disease Control and Prevention (CDC) preterm births - United States 2007. Morbid Mortal Wkly Rep 2011; 60(Suppl): 78-79.

9 Hillier SL, Witkin SS, Krohn MA, Watts DH, Kiviat NB, Eschenbach DA et al. The relationship of amniotic fluid cytokines and preterm delivery, amniotic fluid infection, histologic chorioamnionitis, and chorioamnion infection. Obstet Gynecol 1993; 81(6): 941-948.

10 Andrews WW, Goldenberg RL, Faye-Peterson 0, Cliver S, Goepfert AR, Hauth JC et al. The Alabama preterm birth study: polymorphonuclear and mononuclear cell placental infiltrations, other markers of inflammation, and outcomes in 23-32-week preterm newborn infants. Am J Obstet Gynecol 2006; 195(3): 803-808.

11 Roberts D, Dalziel SR. Antenatal corticosteroids for accelerating fetal lung maturation for women at risk of preterm birth. Cochrane Pregnany and Childbirth Group. Cochrane Database Syst Rev 2010; (6): CD004454

12 Goldenberg RL, Andrews WW, Faye-Peterson OM, Cliver SP, Goepfert AR, Hauth JC et al. The Alabama preterm birth study: corticosteroids and neonatal outcomes in 23- to 32week newborns with various markers of intrauterine infection. Am J Obstet Gynecol 2006; 195(4): 1020-1024. 